\title{
Teaching Professionalism in Surgery and Beyond
}

Joseph Sherman Solomkin

College of Medicine, University of Cincinnati, USA

Correspondence to: Prof. Joseph Solomkin, 231 Albert B. Sabin Way, Cincinnati OH 45267-0558, USA. Email: solomkjs@ucmail.uc.edu

Ann Afr Surg. 2017;14(1):1-2. DOI: https:// dx.doi.org /10.4314/aas.v14i1.1 (C) 2017 Annals of African Surgery. This work is licensed under the Creative Commons Attribution 4.0 International License.

Many people are "professionals" at what they do. They know a wide range of facts and techniques, and have an understanding of the appropriate processes for accomplishing their tasks. This technical knowledge is expected as a minimum requirement of employment, and could be accepted as the definition of "professionalism". More recently however, the meaning of the term "professionalism" has been expanded to include more human activities such as teaching and practicing humility, hope, and humanity with our colleagues and patients (1).

The history of this shift as an organized movement began in 2002, when the US Accreditation Council on Graduate Medical Education (ACGME) mandated that all physicians carry out professional responsibilities that adhered to ethical principles coupled with sensitivity to diverse patient populations. The six ACGME competencies (patient care, medical knowledge, practice-based learning and improvement, interpersonal and communication skills, professionalism as a human dimension, and system-based practice) are now the cornerstone of training and practice for students and practitioners at all levels of health care, and indeed all people working in clinical practice settings.

In the United States, Mark S. Hochberg and his colleagues in the Department of Surgery at NYU School of Medicine are leaders in this area. The curriculum that they have developed, known by its acronym SPICE (Surgical Professionalism and Interpersonal
Communication Education), was incorporated into the American Board of Surgery's SCORE national resident curriculum. The key elements of this program are:

1. Advanced Communication Skills for Surgical Practice-How the Pros Do It

2. Team Respect-Working with Professionals Around You

3. Admitting Mistakes-Ethical Choices and Reasoning

4. Delivering Bad News-Your Chance to Become a Master Surgeon

5. Cultural Diversity-Working Through the Barriers of Language and Differing Customs

6. The Stress of Surgical Practice-The Signs of Stress, Depression, and Suicide in you and Your Surgical Colleagues

7. Managing Your Career-The Professional and Personal Choices That Will Define you and Your Career

Developing the skills, knowledge, and attitudes leading to all of the above goals requires the individual to assume personal responsibility for the care of individual patients, which can have a significant impact on patient safety; a substantial proportion of adverse intraoperative events are attributed to failures in nontechnical skills (2). These authors have well argued that nontechnical skills should be an explicit, integral part of a robust education curriculum to prepare surgeons for safety-focused practice, and ultimately, to reduce surgical errors and other sentinel events attributable to nontechnical skills. 
To further this education effort, they devised a one-day curriculum for surgical residents, which focused on situation awareness, decision-making, communication and teamwork, and leadership (2-4). The effect of the course was defined by responses to a pre-/postquestionnaire. In communication and teamwork, the scores went from an average of $68 \%$ to $78 \%$ (approximate). The true assessment of the effect of this program, improved patient outcomes through better communication at a minimum, was not evaluated. The authors stated that the program was a success. There are considerable difficulties in believing that this very brief encounter will result in the changes that would significantly impact the safety culture of the hospital and all of its components and advocacy groups. It is hard for me to believe that simply explaining to surgical residents (in this case, in a six-hour program) the need for respect and improved communication with nonphysician staff and patients will have the same impact as a more focused approach to organizational culture change. "Change in organizational culture" is a term that encapsulates not only what the members of the organization have learned but also what they believe. It comprises perceptions as well as practices shared within the organization, rather than being solely based on values held by individual members (3). In this issue, the paper by Ojuka et al highlights the aspects of professionalism doctors at an African centre hold dear. Inadequacies in the current curriculum and not having shared values were identified as some of the hindrances to inculcating professionalism (4).

Interventions from an outside country that fails to consider local organization will also likely fail to have any lasting effect. The translation of culture change programs from high income countries with organizational cultures that support specific practices is very likely to be doomed to restricted institutional successes in lower income countries (5). I am further concerned that CUSP-like programs (clinical unit safety programs) are not feasible outside of major academic and sponsor-affiliated hospitals in subSaharan Africa (6). This approach requires assembly of a multidisciplinary team and follows iterative steps of training in the science of safety, identifying patient safety hazards, partnering with senior executives, learning from defects, and implementing tools to improve teamwork and communication. My experience in government level 3 and 4 hospitals in sub-Saharan Africa suggests strongly that teamwork, non-hierarchal communication, and the other personnel resource requirements simply are not present. The article by Musau highlights the issue of dishonesty in medical training that could explain the lack of professionalism in the graduates of such a system (7). The paper also reveals that our high schools might be the breeding grounds for dishonesty and this might be an area to target.

\section{References}

1. Hochberg MS, Berman RS, Pachter HL. Professionalism in Surgery: Crucial Skills for Attendings and Residents. Adv Surg. 2017; 51: 229-49.

2. Lin Y, Scott JW, Yi S, et al. Improving Surgical Safety and Nontechnical Skills in Variable-Resource Contexts: A Novel Educational Curriculum. J Surg Educ. 2017. pii: S1931-7204(17)30483-X.

3. De Bono S, Heling G, Borg MA. Organizational Culture and its Implications for Infection Prevention and Control in Healthcare Institutions. J Hosp Infect. 2014; 86(1):1-6.

4. Ojuka DK, Olenja J, Mwango'mbe $\mathrm{N}$, et al. Nurturing Medical Professionalism in the Surgical Community. Ann Afr Surg. 2017;14(2): 10-18.

5. Gould DJ, Hewitt-Taylor J, Drey NS, et al. The CleanYourHandsCampaign: Critiquing Policy and Evidence Base. J Hosp Infect. 2007 Feb;65(2): 95101.

6. WickEC, GalanteDJ, Hobson DB, etal. Organizational Culture Changes Result in Improvement in Patient-Centered Outcomes: Implementation of an Integrated Recovery Pathway for Surgical Patients. J Am Coll Surg. 2015 Sep;221(3):66977; quiz 785-6.

7. Musau P, Academic Dishonesty in Medical Schools. Ann Afr Surg. 2017;14(1): 19-21. 